\title{
Attractive versus Repulsive Excitonic Interactions of Colloidal Quantum Dots Control Blue- to Red-Shifting (and Non-shifting) Amplified Spontaneous Emission
}

\author{
Ahmet Fatih Cihan, ${ }^{\dagger, \|, \perp}$ Yusuf Kelestemur, ${ }^{\dagger, \|}$ Burak Guzelturk, ${ }^{\dagger, \dagger}$ Ozan Yerli, ${ }^{\dagger}$ Ulas Kurum, ${ }^{\S}$ \\ Halime Gul Yaglioglu, ${ }^{\S}$ Ayhan Elmali, ${ }^{\S}$ and Hilmi Volkan Demir*, ${ }^{*},+$ \\ ${ }^{\dagger}$ Department of Electrical and Electronics Engineering, Department of Physics, UNAM-Institute of Materials Science and \\ Nanotechnology, Bilkent University, Ankara 06800, Turkey \\ ${ }^{\ddagger}$ School of Electrical and Electronic Engineering, School of Physical and Mathematical Sciences, Nanyang Technological University, \\ Nanyang Avenue, Singapore 639798, Singapore \\ ${ }^{\S}$ Department of Engineering Physics, Ankara University, Ankara 06100, Turkey
}

Supporting Information

ABSTRACT: Tunable, high-performance, two-photon absorption (TPA)-based amplified spontaneous emission (ASE) from near-unity quantum efficiency colloidal quantum dots (CQDs) is reported. Besides the absolute spectral tuning of ASE, the relative spectral tuning of ASE peak with respect to spontaneous emission was shown through engineering excitonic interactions in quasi-type-II CdSe/CdS core/shell CQDs. With core-shell size adjustments, it was revealed that Coulombic exciton-exciton interactions can be tuned to be attractive (type-I-like) or repulsive (type-II-like) leading to red- or blue-shifted ASE peak, respectively, and that nonshifting ASE can be achieved with the right core-shell combinations. The possibility of obtaining ASE at a specific wavelength from both type-Ilike and type-II-like CQDs was also demonstrated. The experimental observations were supported by parametric quantum-mechanical modeling, shedding light on the typetunability. These excitonically engineered CQD-solids exhibited TPA-based ASE threshold as low as $6.5 \mathrm{~mJ} / \mathrm{cm}^{2}$ under $800 \mathrm{~nm}$ excitation, displaying one of the highest values of TPA cross-section of $44660 \mathrm{GM}$.
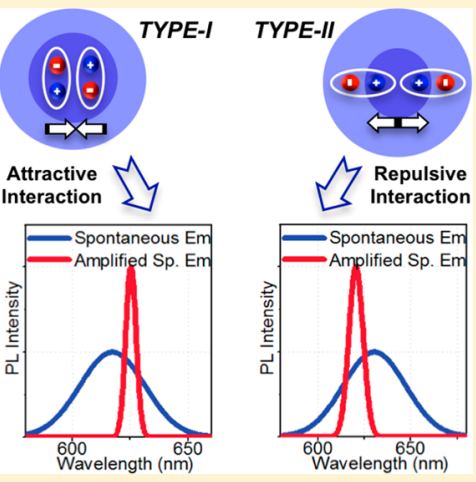

SECTION: Physical Processes in Nanomaterials and Nanostructures

$\mathrm{O}$ wing to their size tunable and favorable optical properties, semiconductor colloidal quantum dots (CQDs), also known as nanocrystals, have become highly attractive as light-generating materials for light-emitting diodes $^{1-3}$ and as optical gain media for lasing applications., ${ }^{4,5}$ Strong quantum confinement effects observed in CQDs lead to several advantages over their bulk-counterparts including enhanced exciton binding energies and efficient band edge emission at room temperature due to the increased charge carrier concentration in small CQD volumes. CQDs also allow for engineering of their electronic properties via tuning of their size, $^{2}$ shape (spherical, nanorod-shaped ${ }^{6}$ and tetrapod-shaped ${ }^{7}$ ) and excitonic characteristics resulting in distinct electronic behavior, namely, type-I, type-II, ${ }^{8}$ or quasi-type-II. ${ }^{9}$ Consequently, CQDs have arisen as a promising class of lasing materials offering lower optical gain thresholds, higher temperature insensitivity, ease of processability via facile wetchemistry methods, and ease of emission tunability covering a wide spectral range. ${ }^{10-12}$ These attributes make CQDs appealing as compared to other emerging material systems including organics and epitaxially templated quantum-confined systems.
Optical gain performances of various CQDs have previously been studied and relatively low optical gain thresholds have been reported with respectable temperature stabilities and narrow emission bandwidths. ${ }^{13}$ Optically pumped CQD lasers have been realized using various photonic structures (e.g., in optical cavities made up of distributed Bragg reflectors, above grating structures providing distributed feedback). ${ }^{14-18}$ In these previous studies, single-photon absorption mechanism was utilized for optical pumping. Also, in few recent studies, as an alternative to single-photon optical pumping, two-photon absorption (TPA) mechanism ${ }^{19-22}$ was employed to realize amplified spontaneous emission (ASE) in CQDs, which offers a lower risk of photodamaging the sample. This is also advantageous because CQDs exhibit very high TPA cross sections. ${ }^{23,24}$ Among these previously studied CQD systems are $\mathrm{CdSe} / \mathrm{CdS} / \mathrm{ZnS}$ core/shell/shell CQDs ${ }^{19-22}$ and $\mathrm{CdSe} / \mathrm{CdS}$ nanorods. $^{25}$

Received: October 13, 2013

Accepted: November 21, 2013

Published: November 21, 2013 

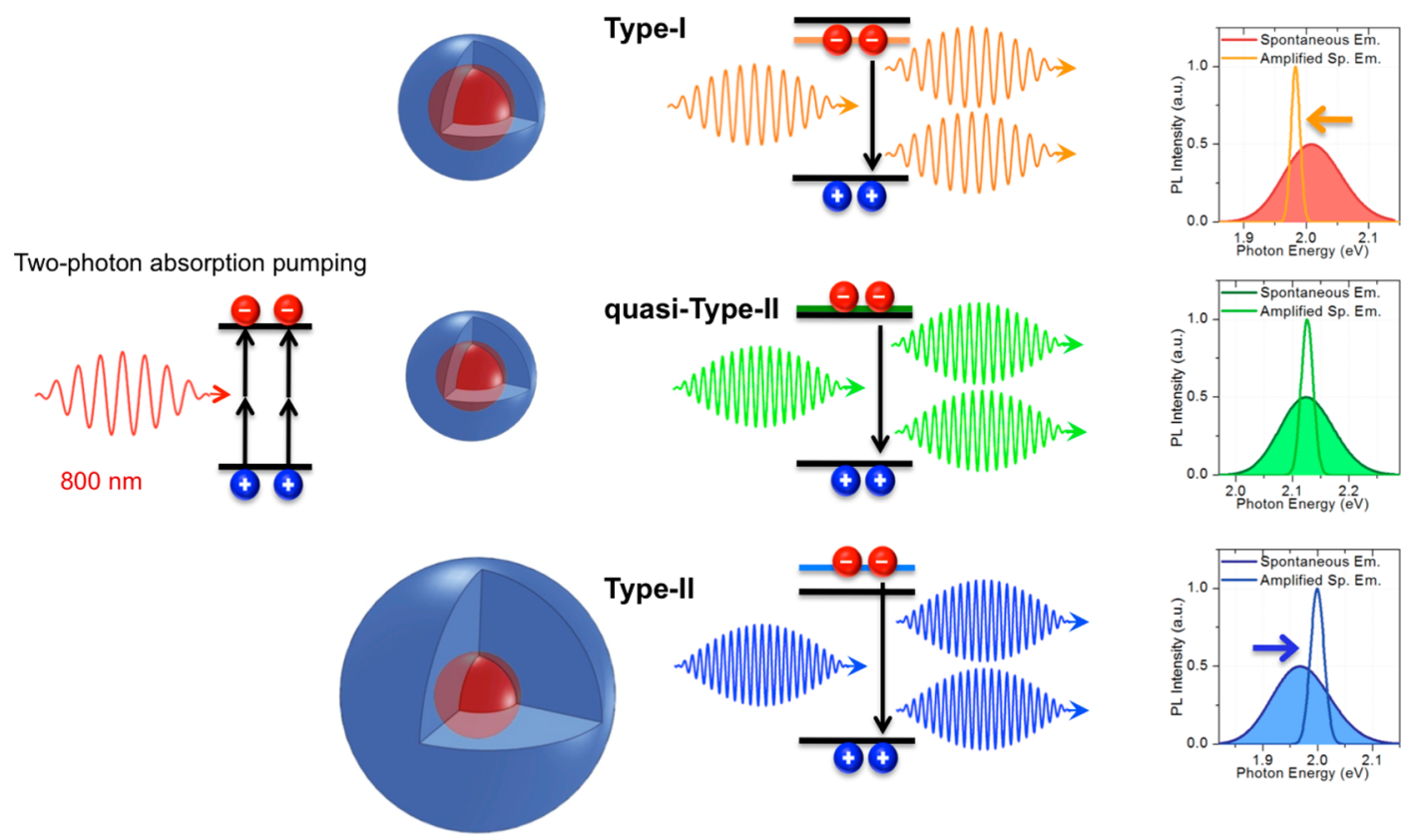

Figure 1. Schematics of the TPA-pumping of quasi-type-II CdSe/CdS core/shell CQDs and relative tuning of their ASE via type-tuning of these CQDs by core/shell dimension adjustments resulting in red- to blue-shifted ASE peak with respect to spontaneous emission. Also, note the nonshifting ASE between the red- and blue-shifting regimes.

As a unique and fundamental feature of core/shell and multishell CQDs, the core-shell size adjustments provide us with the ability to control the nature and strength of excitonexciton $(\mathrm{X}-\mathrm{X})$ interactions by tuning electron and hole wave function localizations across the core and the shell. Therefore, the repulsive (type-II-like) versus attractive (type-I-like) nature of $\mathrm{X}-\mathrm{X}$ interactions can be engineered to modify optical and electronic properties. ${ }^{26-28}$ Within this context, Cooney et al. has recently shown tunable ASE from $S$ and $P$ states of the CQDs via state-resolved pumping, ${ }^{29}$ but the potential of the electronic-type engineering of the CQDs for lasing applications was not considered. In the work of Saba et al., in seeded CdSe/ $\mathrm{CdS}$ core/shell nanorods, tuning of biexciton emission was demonstrated by shifting the electronic structure from type-I to quasi-type-II. ${ }^{30}$ Although, in principle, quasi-type-II CQDs offers the potential to operate in both regimes, ${ }^{31}$ to date, to the best of our knowledge, no single material system has been shown to exhibit ASE tunability from type-I-like to type-II-like behavior, i.e., red- to blue-shifted ASE with respect to spontaneous emission, respectively. This implies that it should be in fact possible, with the right CQD core-shell design, to achieve also nonshifting ASE, which has not been previously achieved. Mastering $\mathrm{X}-\mathrm{X}$ interactions and developing full control over the ASE are important for performance improvements of CQD lasing systems by enabling the engineering and optimization of gain/loss mechanisms in these systems.

In this study, utilizing quasi-type-II $\mathrm{CdSe} / \mathrm{CdS}$ core/shell CQDs and studying their ASE systematically under two-photon excitation, we present the first account of absolute and relative tuning of the ASE characteristics going from type-I-like to typeII-like regimes in a single material system, as pictorially illustrated in Figure 1. These CQDs are particularly chosen for this study, as they offer near-unity quantum efficiency (QE) with significantly suppressed Auger recombination, as a result of the partial separation of electron and hole wave functions within the core-shell structure. ${ }^{13,32-34}$ The TPA cross-section of these CQDs, measured by $z$-scan technique at $800 \mathrm{~nm}$ excitation wavelength, was found to be ca. 44660 GM which is one of the highest reported values when compared to the CQDs of similar size. This resulted in the observation of a very low optical gain threshold value of $6.5 \mathrm{~mJ} / \mathrm{cm}^{2}$ per pulse at 800 $\mathrm{nm}$ excitation wavelength without any loss compensation or waveguiding mechanism. Although the experimental conditions are not exactly the same, this threshold value is reasonably low compared to the previous works of Zhang et al. $\left(6.2 \mathrm{~mJ} / \mathrm{cm}^{2}\right.$ threshold of samples of larger CdSe/CdS/ZnS CQDs employing a waveguiding mechanism $)^{22}$ and Jasieniak et al. (10.4 mJ/ $\mathrm{cm}^{2}$ threshold of larger $\mathrm{CdSe} / \mathrm{CdS} / \mathrm{ZnS}$ CQDs with a waveguiding mechanism). ${ }^{19}$ Using these CdSe/CdS CQDs, the tunability of the ASE peak with respect to spontaneous emission was achieved via carefully engineering their $\mathrm{X}-\mathrm{X}$ interactions by changing the core size and shell thickness of the CQDs. Here we showed that a single material system can exhibit full ASE tuning variation from blue- to red-shifting behavior, and nonshifting ASE is possible between these two regimes. The ASE peak shift we obtained for CdSe/CdS CQDs in the blue-shifting regime is $32 \mathrm{meV}$ (about $10 \mathrm{~nm}$ ), while it is ca. $25 \mathrm{meV}$ (about $8 \mathrm{~nm}$ ) in the red-shifting regime. Also, we demonstrated that there is no unique core-shell combination to obtain certain ASE characteristics and that it is possible to obtain ASE at a specific wavelength of choice within the feasible range from both type-I-like and type-II-like excitonically engineered $\mathrm{CdSe} / \mathrm{CdS} \mathrm{CQDs}$. These experimental results were supported by parametrized quantum mechanical modeling of the electronic structure of the CQDs for various core sizes and shell thicknesses. We believe that, with all of these attractive properties, $\mathrm{CdSe} / \mathrm{CdS} \mathrm{CQDs}$ make very strong alternatives to the widely studied $\mathrm{CdSe} / \mathrm{ZnS} \mathrm{CQDs}$ for lasing applications. $^{18,35,36}$

Highly efficient CdSe/CdS core/shell CQDs were synthesized with the selective ion layer adsorption and reaction (SILAR) technique as described in the literature. ${ }^{37}$ With these CQDs having a core diameter in the range of $2.2-2.4 \mathrm{~nm}$ and an average shell thickness in the range of 2.4-3.2 monolayers, 

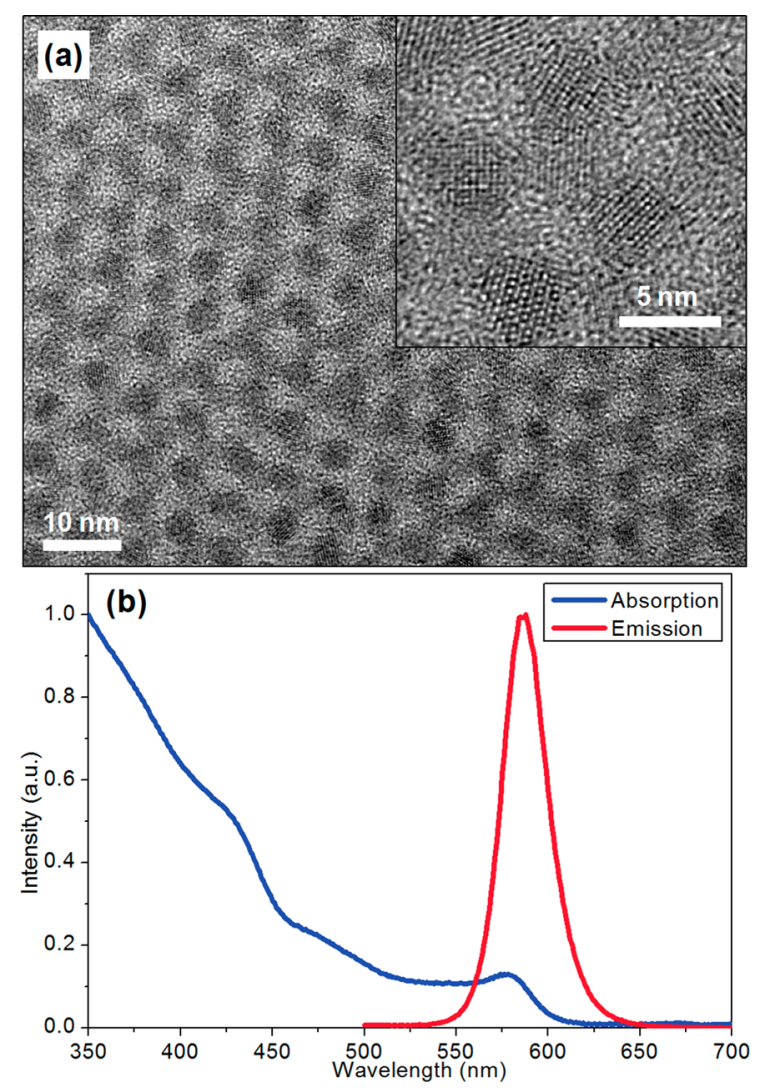

Figure 2. (a) High resolution TEM images and (b) PL-absorption spectra of CdSe/CdS CQDs.

whose steady-state photoluminescence (PL) and absorption spectra are given in Figure 2b, a high QE level of $90-95 \%$ was achieved. An important feature of this $\mathrm{CdSe} / \mathrm{CdS}$ material system is the very small lattice mismatch (3.9\%) between CdSe and $\mathrm{CdS}$, which results in the synthesis of potentially defectfree CQDs with near-unity QE. Moreover, as can be seen from the transmission electron microscopy (TEM) image in Figure $2 \mathrm{a}$, nearly perfect arrangement of the CQDs indicates that they have a very narrow size distribution with a high degree of crystallinity of the structure.

Obtaining a high TPA cross-section is very critical for achieving low-threshold TPA-based optical gain because of the direct correlation between the TPA cross-section and the number of absorbed photons, which determines the onset of population inversion. Therefore, we conducted open-aperture $z$-scan measurements of $\mathrm{CdSe} / \mathrm{CdS} \mathrm{CQDs}$ having a core diameter of $3.2 \mathrm{~nm}$ and shell thickness of $1.1 \mathrm{~nm}$ (see the Supporting Information for the $z$-scan measurement results and analysis). We found the TPA cross-section value to be $\sigma_{2}=$ $44660 \mathrm{GM}$, which is very high compared to CdSe/ZnS CQDs of similar sizes. ${ }^{19}$ Hence, CdSe/CdS CQDs stand out as promising two-photon sensitizers. This higher TPA crosssection of $\mathrm{CdSe} / \mathrm{CdS} \mathrm{CQDs}$ compared to $\mathrm{CdSe} / \mathrm{ZnS}$ can be explained by the increased contribution coming from the $\mathrm{CdS}$ shell having a band gap of $2.5 \mathrm{eV}$ (see Figure $2 \mathrm{~b}$ for the absorption of $\mathrm{CdSe} / \mathrm{CdS} \mathrm{CQDs}$ exhibiting a strong kink around $430 \mathrm{~nm}$ due to the absorption of the CdS shell), while the $\mathrm{ZnS}$ shell has a too large band gap $(3.9 \mathrm{eV})$ that it cannot contribute to the TPA at $800 \mathrm{~nm}(1.55 \mathrm{eV}) .^{38}$

ASE performance of highly concentrated drop-casted solid films of CdSe/CdS CQDs (see Methods for the sample preparation) was investigated through excitation intensity dependent PL emission measurements, under a pulsed excitation at $800 \mathrm{~nm}$ with a $45 \mathrm{fs}$ pulse-width, as shown in Figure 3a. As can be seen here, when the excitation intensity
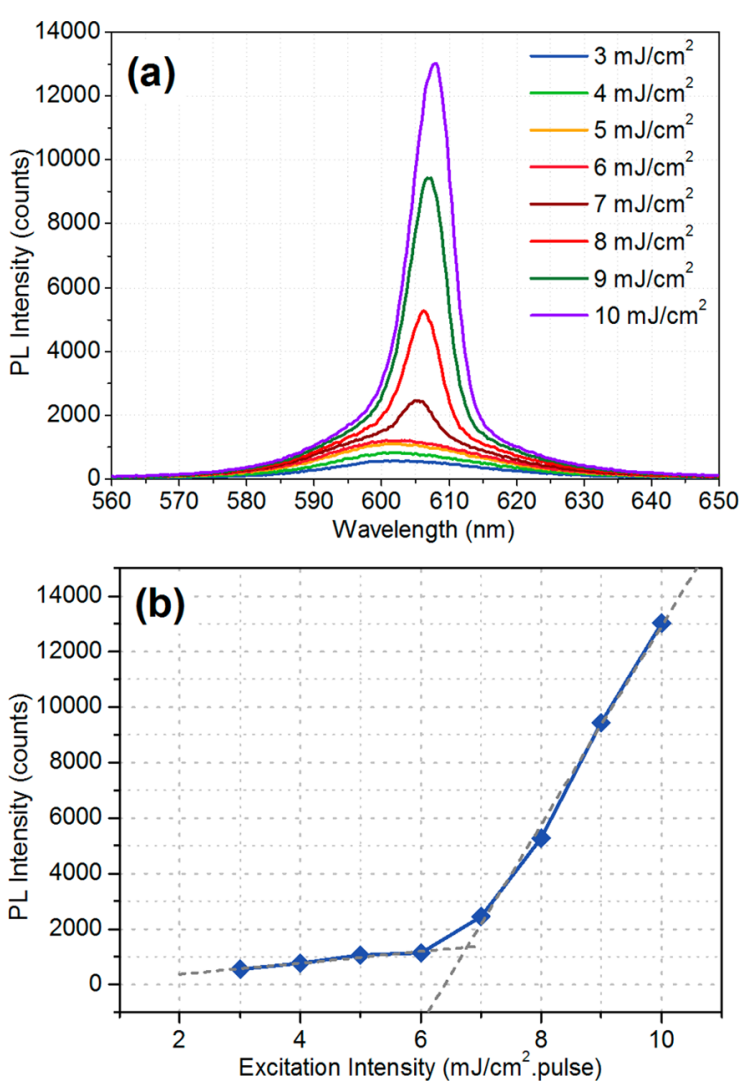

Figure 3. (a) Evolution of the PL spectra of CdSe/CdS CQDs with varying excitation intensity. (b) Excitation pulse intensity dependence of the PL intensity at the wavelength of the ASE process. The linear fits shown as dashed lines are guides to the eye about the slopes of the curves.

exceeded the optical gain threshold, ASE peak emerged at the ca. $3 \mathrm{~nm}(11 \mathrm{meV})$ longer wavelength side of the spontaneous emission peak. The full width at half-maximum (fwhm) of the ASE and the spontaneous emission peaks of the sample excited with an intensity of $10 \mathrm{~mJ} / \mathrm{cm}^{2}$ per pulse were obtained as 5.7 and $21.4 \mathrm{~nm}$ (19 and $73 \mathrm{meV}$ ), respectively, by fitting the PL spectrum with two Gaussian functions. The spectral integration of these two Gaussian functions indicated that, although it is spectrally very narrow, the emission due to the ASE process accounted for more than $50 \%$ of all the emission from the sample in the direction of collection. As shown in Figure 3b, the excitation intensity threshold for ASE is ca. $6.5 \mathrm{~mJ} / \mathrm{cm}^{2}$ per pulse. The ASE performance of this sample can be said to be very good considering the fact that there is no loss compensation or wave-guiding effect favoring the optical gain in the sample. The concomitant spontaneous emission is due to scattering and diffusion of the excitation light, resulting in outof-focus excitation of the sample.

In terms of spectral behavior of the ASE process as depicted above, $3 \mathrm{~nm}$ red-shifted ASE was observed with respect to spontaneous emission, whereas this spectral difference is expected to be around $15-20 \mathrm{~nm}$ in regular type-I CQDs. ${ }^{19}$ This shows that the structure is in the quasi-type-II regime, but it is closer to being type-I-like rather than being type-II-like. In 
(a)

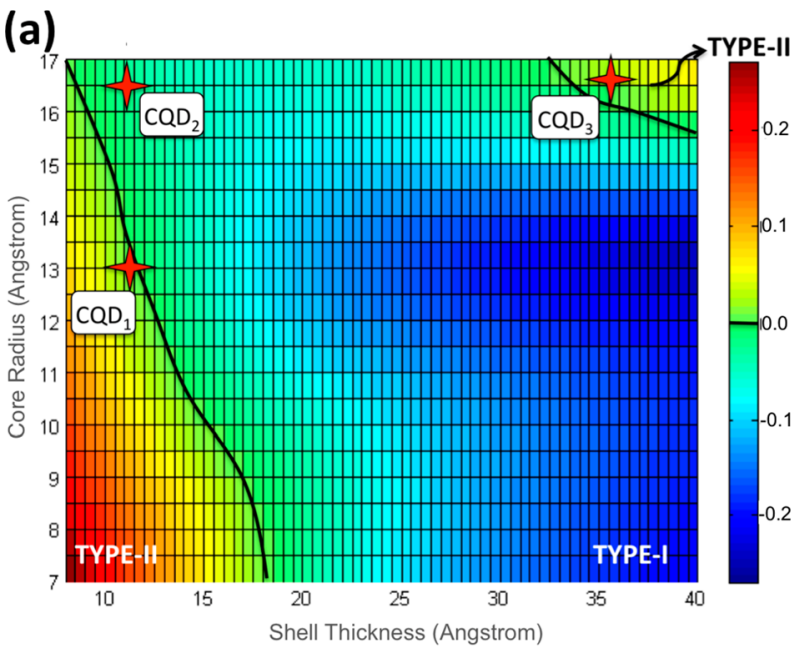

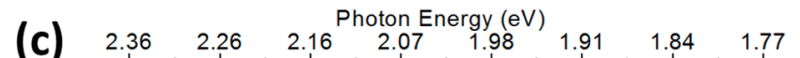

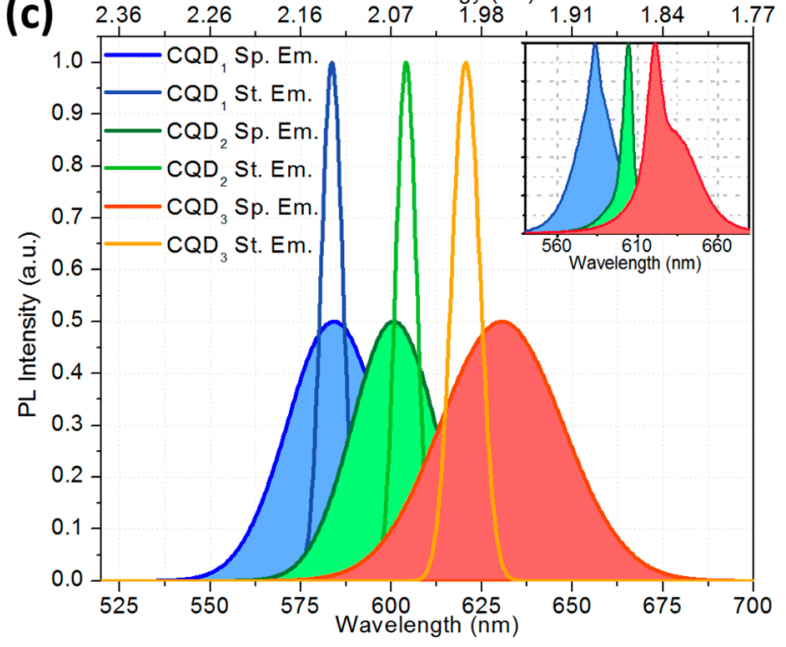

(b)
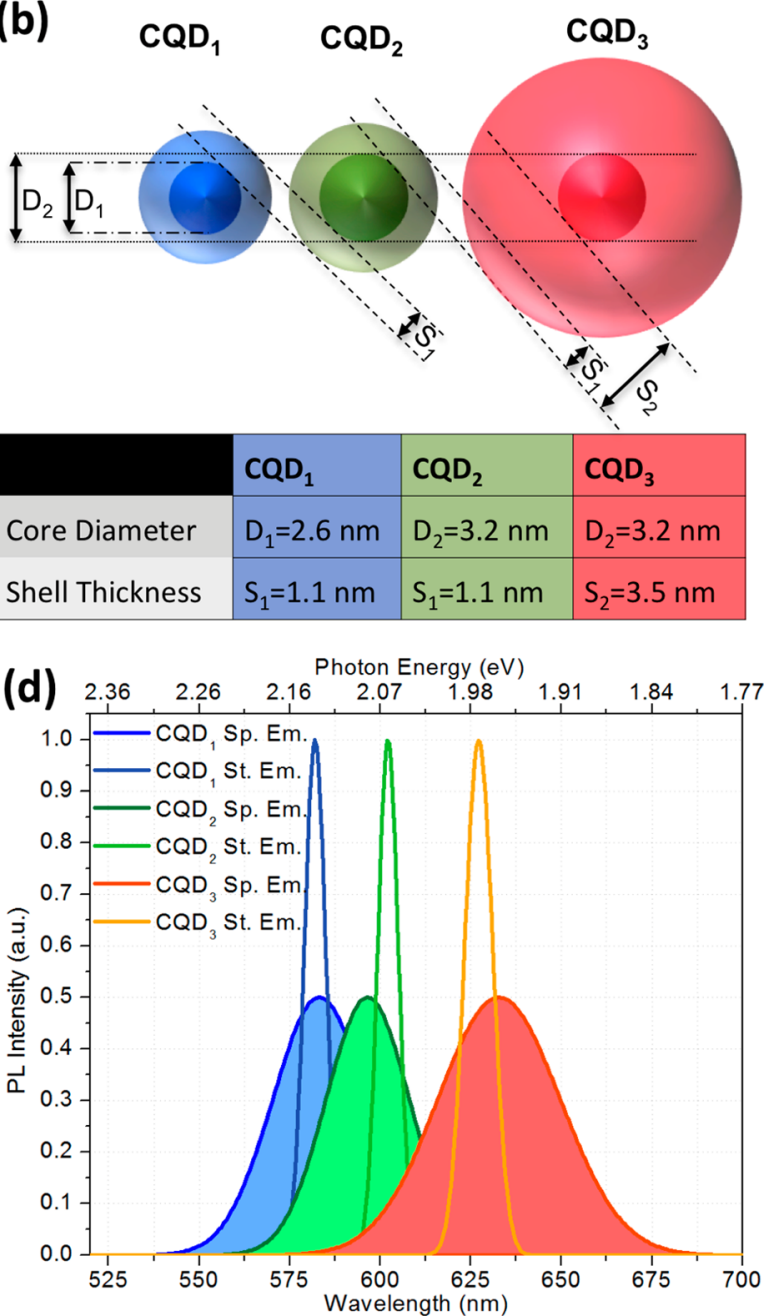

Figure 4. (a) ASE peak shift (or BX peak shift) with respect to spontaneous emission peak (or X peak) of core/shell CQDs in eVs calculated for varying core radius and shell thickness. The black lines correspond to zero shift values, that is, the transition dimensions from type-I to type-II behavior. The red stars show the corresponding positions of $C Q D_{1}, C Q D_{2}$, and $\mathrm{CQD}_{3}$, whose ASE spectra are provided in parts (c) and (d), of the figure. (b) Schematics of CQDs where the dimensions are proportional to their real dimensions. Dashed lines are provided to compare the core diameters and shell thicknesses of the CQDs. Table shows the core diameters and shell thicknesses of CQDs whose experimental and theoretical emission spectra are given in $(c)$ and $(d)$. Note that $C Q D_{1}$ and $C Q D_{2}$ have the same shell thickness while $C Q D_{2}$ and $C Q D_{3}$ have the same core radius. (c) Normalized decomposed ASE (or stimulated emission (St. Em.)) and spontaneous emission (Sp. Em.) spectra of three different CQDs under intense two-photon excitation conditions. The under-filled spectra, which are normalized to 0.5 au, are the spontaneous emission spectra of the CQDs, while the narrow spectra normalized to 1 are the ASE spectra of the CQDs. The inset shows the original experimental PL spectra of CQDs without decomposition. (d) Normalized decomposed ASE and spontaneous emission spectra of three different CQDs obtained by quantum mechanical simulations.

other words, $3 \mathrm{~nm}$ shift implies that the local charge neutrality is distorted in these CQDs as observed in type-II structures; ${ }^{39}$ however, the distortion is not strong enough to give rise to a significant repulsive $\mathrm{X}-\mathrm{X}$ interaction in the CQDs.

To understand and demonstrate the type-tunability of ASE mechanism, we conducted parametric biexciton (BX) and single exciton (X) energy calculations for physically meaningful ranges of core and shell sizes by using effective mass approximation on self-consistent and iterative Kohn-Sham equation solutions (see the Supporting Information for the details of the simulations). The resulting energy difference between BXs and Xs are provided in Figure 4a with respect to the core radius and shell thickness. Since ASE occurs from BX states in the CQDs used in this study at the reported excitation levels, the spectral positions of BXs and Xs correspond to the spectral positions of the ASE and the spontaneous emission, respectively. Therefore, the corresponding core/shell sizes of CQDs for type-I-like and type-II-like behaviors are clearly visible on this plot, where the color bar shows the $\mathrm{BX}-\mathrm{X}$ spectral difference in eVs.

To experimentally demonstrate the type-tunability feature of the CdSe/CdS CQDs from type-I-like to type-II-like behaviors, three different $\mathrm{CdSe} / \mathrm{CdS}$ core/shell CQDs having different core diameters and shell thicknesses were synthesized. The corresponding positions of these are indicated with red-stars in Figure 4a. The core/shell sizes of these samples were chosen to obtain repulsive, attractive, and neither repulsive nor attractive $\mathrm{X}-\mathrm{X}$ interactions corresponding to type-II, type-I, and quasitype-II regimes, respectively. The room temperature normalized PL spectra of the three CdSe/CdS CQDs, whose size information is provided in Figure $4 \mathrm{~b}$, under intense two-photon optical excitation are given in the inset of Figure $4 c$ as a 
demonstration of relative tunability of ASE. (Note that the CQDs whose PL spectra are provided in this figure do not necessarily have near-unity $\mathrm{QE}$ since it is not possible to obtain near-unity QE for all CQD sizes. Near-unity QE CQDs used in the ASE performance evaluation experiments were discussed previously.) The spontaneous emission and the ASE contributions in the total PL emissions of these samples were extracted by fitting the PL spectra with two Gaussian distribution functions and provided in Figure 4c. ASE peak is clearly blue-shifted compared to the spontaneous emission peak in $\mathrm{CQD}_{3}$ sample (red curve), while it is red-shifted for the $\mathrm{CQD}_{2}$ sample (green curve). However, ASE peak is almost at the same position as the spontaneous emission of $\mathrm{CQD}_{1}$ sample (blue curve). The reason for the blue-shifted ASE peak in $\mathrm{CQD}_{3}$ is that these CQDs have a very thick shell, which allows the electrons to delocalize freely over the entire CQD volume, while the holes are confined in the core, which results in type-II-like carrier distributions in these CQDs. It is shown in the literature that because of the repulsive $\mathrm{X}-\mathrm{X}$ Coulomb interactions in type-II CQDs, the $\mathrm{BX}$ recombination event takes place at higher photon energies. ${ }^{40}$ Since the ASE process occurs from BX states in these samples, as schematically shown in Figure 1, it is blue-shifted in $\mathrm{CQD}_{3}$ sample. On the other hand, the situation is reversed in $C Q D_{2}$ sample where the core is big enough to accommodate both electrons and holes resulting in type-I-like carrier distributions, and hence an attractive $\mathrm{X}-\mathrm{X}$ interaction. From the peak overlap of ASE and spontaneous emission events in $\mathrm{CQD}_{1}$ sample, we can say that the carrier distributions in these CQDs exhibit a quasi-type-II behavior, which is the transition regime between type-I and type-II. Therefore, ASE peak is neither blue- nor red-shifted in the $\mathrm{CQD}_{1}$ sample.

The physical origin of the relative tunability of spectral position of ASE peak with respect to spontaneous emission peak for this material system is the low energy barrier of $0.3 \mathrm{eV}$ for electrons at the core/shell interface in CdSe/CdS CQDs. Because of this low energy barrier for electrons, it is difficult to totally confine the electrons in the core, while the holes are more strongly confined in the core thanks to their larger effective mass and higher energy barrier of $0.78 \mathrm{eV} .^{41}$ The only way of confining the electrons in the core is making the core big enough and the shell thin enough, as it is the case for $\mathrm{CQD}_{2}$ sample. Being able to adjust the overlap of electron and hole wave functions means that CQDs can be made type-I-like (where both electrons and holes are confined to the same part of the structure) or type-II-like (where electrons and holes are confined to different parts of the structure). Therefore, ASE peak can be manipulated through controlling the BX energies, which have intrinsically opposite signs for type-I and type-II systems such that the ASE peak can be chosen to be either on the red or blue side of the PL peak of the CQDs. ${ }^{42}$ This can provide us with the opportunity to optimize the efficiency of stimulated emission in CQDs by deciding whether optical gain would occur from single exciton states or BX states. ${ }^{8}$ Furthermore, the relative adjustability of ASE peak position can enable us to suppress the loss mechanism of reabsorption of the photons that are supposed to contribute to the optical gain process. $^{40}$

In addition to the experimental results provided in Figure 4c, the calculated ASE and spontaneous emission peaks for the dimensions of these three CQD samples are provided in Figure $4 \mathrm{~d}$ where the well-agreement with the experimental results in Figure $4 \mathrm{c}$ is apparent. To obtain the spectra in Figure $4 d$, we calculated the $\mathrm{X}$ and $\mathrm{BX}$ energies for the average $\mathrm{CQD}$ sizes for the three samples and plotted Gaussian functions centered at the calculated $\mathrm{X}$ and $\mathrm{BX}$ energies (The spectral shifts are also available in Figure 4a). Here note that the spectral widths of these Gaussian functions were taken the same as the experimental measurements to account for the inhomogeneous broadening caused by the size distribution of the CQD ensemble.

To verify the claim that one can obtain either type-I-like or type-II-like ASE behavior at a specific wavelength from $\mathrm{CdSe} /$ CdS CQDs by means of this kind of $\mathrm{X}-\mathrm{X}$ interaction engineering, we conducted ASE experiment on another nearunity QE CQD sample whose PL spectra under high intensity pumping is provided in Figure 5a together with the spectra of
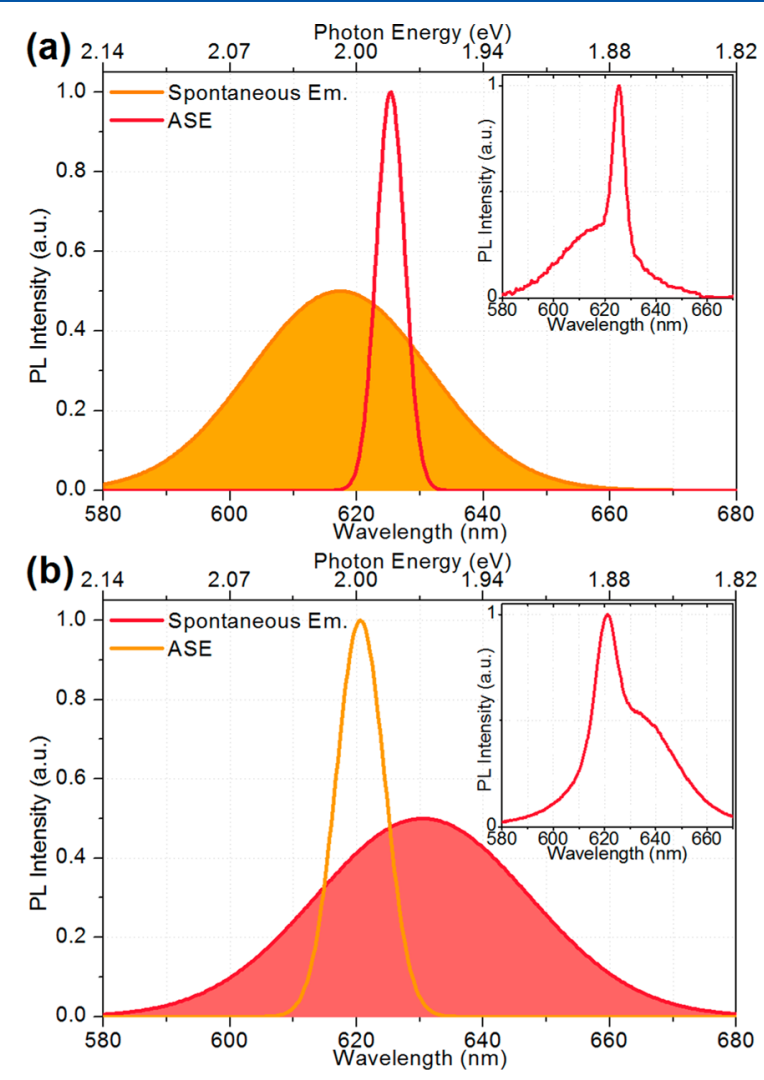

Figure 5. Normalized decomposed experimental ASE and spontaneous emission spectra of (a) near-unity QE CQDs and (b) $\mathrm{CQD}_{3}$ sample under intense two-photon excitation conditions. The underfilled spectra, which are normalized to $0.5 \mathrm{au}$, are the spontaneous emission spectra of the CQDs, while the narrow spectra normalized to 1 are the ASE spectra of the CQDs. The insets show the original experimental PL spectra of CQDs without decomposition.

$\mathrm{CQD}_{3}$ sample in Figure $5 \mathrm{~b}$ for comparison. As can be seen there, ASE peaks of the samples arise almost at the same spectral position although their spontaneous emission peaks are on the red and blue side of their ASE peaks. This result shows that it is possible to obtain blue- or red-shifted ASE behavior at a specific spectral position from these CQDs, which would be a crucial tool for the CQD-based single or multicolor lasing applications.

In conclusion, we demonstrated a high performance typetunable ASE from near-unity QE CdSe/CdS core/shell CQDs excited via a two-photon excitation mechanism. The TPA crosssection of $\mathrm{CdSe} / \mathrm{CdS} \mathrm{CQDs}$ is found to strongly support the 
claim that these CQDs are promising for TPA applications. The absolute and relative spectral tunabilities of ASE for type-I-like, type-II-like, and quasi-type-II operations (adjustable ASE shift from spontaneous emission) from the same material system were, for the first time, shown as other exceptional features of $\mathrm{CdSe} / \mathrm{CdS}$ CQDs, which make them very strong competitors against other emitters for CQD lasing applications. Typetunability of $\mathrm{CdSe} / \mathrm{CdS} \mathrm{CQDs}$ through $\mathrm{X}-\mathrm{X}$ interaction engineering can be considered to be a promising method for optimizing the lasing performances of future CQD lasers. With proper $\mathrm{X}-\mathrm{X}$ interaction engineering, it is possible to obtain ASE at a certain wavelength on the lower or higher energy side of the spontaneous emission of CQDs. For future applications, high-performance ASE through TPA process from these CQDs together with their type-tunability feature are good indicators for the feasibility of CQD lasing via two-photon pumping.

\section{EXPERIMENTAL METHODS}

Synthesis of Quantum Dots. The synthesis of CdSe/CdS CQDs, except for the $\mathrm{CQD}_{3}$ sample, which has a very thick shell, was made through modified selective ion layer adsorption and reaction (SILAR) technique as Greytak et al. reported. ${ }^{37,43}$ The details of both synthesis methods are provided in the Supporting Information.

Preparation of Quantum Dot Film Samples. The thin-film samples of CQDs were prepared by drop-casting of highly concentrated (ca. $50 \mathrm{mg} / \mathrm{mL}$ ) solutions of CQDs dissolved in hexane on $1 \mathrm{~cm}$ by $1 \mathrm{~cm}$ quartz substrates. Then the solvent was allowed to slowly evaporate at room temperature leaving very highly stacked CQD layers on top of the quartz substrates.

Steady-State Photoluminescence Measurements. For ASE experiments, as the excitation source for the CQD samples, we used a femtosecond optical amplifier (Spectra Physics, Spitfire Pro $\mathrm{XP}$ ) with an output beam of $45 \mathrm{fs}$ pulse width at $800 \mathrm{~nm}$ wavelength. The amplifier is seeded by a Ti:Sapphire laser (Spectra Physics, Tsunami). The laser beam is focused to a spot whose area is ca. $1 \mathrm{~mm}^{2}$ on the sample with the help of a thin lens with a $10 \mathrm{~cm}$ focal length. The emission spectra of the samples were collected by an optical fiber connected to a miniature spectrometer (Ocean Optics).

\section{ASSOCIATED CONTENT}

\section{S Supporting Information}

Synthesis and characterization of $\mathrm{CdSe} / \mathrm{CdS}$ core/shell quantum dots, two-photon absorption cross-section measurements, quantum mechanical simulations. This material is available free of charge via the Internet at http://pubs.acs.org.

\section{AUTHOR INFORMATION}

\section{Corresponding Author}

*E-mail: hvdemir@ntu.edu.sg; volkan@bilkent.edu.tr.

\section{Present Address}

${ }^{\perp}$ Department of Electrical Engineering, Stanford University, Stanford, CA 94305, United States.

\section{Author Contributions}

"These authors contributed equally.

\section{Author Contributions}

The manuscript was written through contributions of all authors. All authors have given approval to the final version of the manuscript.

Notes

The authors declare no competing financial interest.

\section{ACKNOWLEDGMENTS}

This work is supported in part by the Singapore National Research Foundation under the program numbers of NRFCRP-6-2010-02 and NRF-RF-2009-09, and in part by ESFEURYI. Also, H.V.D. gratefully acknowledges support from TUBA-GEBIP. A.F.C. and Y.K. acknowledge support from TUBITAK BIDEB.

\section{REFERENCES}

(1) Shirasaki, Y.; Supran, G. J.; Bawendi, M. G.; Bulović, V. Emergence of Colloidal Quantum-Dot Light-Emitting Technologies. Nat. Photon. 2013, 7, 13-23.

(2) Nizamoglu, S.; Zengin, G.; Demir, H. V. Color-Converting Combinations of Nanocrystal Emitters for Warm-White Light Generation with High Color Rendering Index. Appl. Phys. Lett. 2008, 92, 031102.

(3) Coe, S.; Woo, W.-K.; Bawendi, M. G.; Bulović, V. Electroluminescence from Single Monolayers of Nanocrystals in Molecular Organic Devices. Nature 2002, 420, 800-803.

(4) Klimov, V. I.; Mikhailovsky, A. A.; Xu, S.; Malko, A.; Hollingsworth, J. A.; A.Leatherdale, C.; Eisler, H.-J.; G, B. M. Optical Gain and Stimulated Emission in Nanocrystal Quantum Dots. Science 2000, 290, 314-317.

(5) Kambhampati, P. Hot Exciton Relaxation Dynamics in Semiconductor Quantum Dots: Radiationless Transitions on the Nanoscale. J. Phys. Chem. C 2011, 115, 22089-22109.

(6) Krahne, R.; Zavelani-Rossi, M.; Lupo, M. G.; Manna, L.; Lanzani, G. Amplified Spontaneous Emission from Core and Shell Transitions in CdSe/CdS Nanorods Fabricated by Seeded Growth. Appl. Phys. Lett. 2011, 98, 063105.

(7) Liao, Y.; Xing, G.; Mishra, N.; Sum, T. C.; Chan, Y. Low Threshold, Amplified Spontaneous Emission from Core-seeded Semiconductor Nanotetrapods Incorporated into a Sol-Gel Matrix. Adv. Opt. Mater. 2012, 24, 159-164.

(8) Klimov, V. I.; Ivanov, S. a; Nanda, J.; Achermann, M.; Bezel, I.; McGuire, J. A.; Piryatinski, A. Single-Exciton Optical Gain in Semiconductor Nanocrystals. Nature 2007, 447, 441-446.

(9) Kambhampati, P. Unraveling the Structure and Dynamics of Excitons in Semiconductor Quantum Dots. Acc. Chem. Res. 2011, 44, $1-13$.

(10) Kambhampati, P. Multiexcitons in Semiconductor Nanocrystals: A Platform for Optoelectronics at High Carrier Concentration. J. Phys. Chem. Lett. 2012, 3, 1182-1190.

(11) Stewart, J. T.; Padilha, L. A.; Bae, W. K.; Koh, W.; Pietryga, M.; Klimov, V. I. Carrier Multiplication in Quantum Dots Within the Framework of Two Competing Energy Relaxation Mechanisms. J. Phys. Chem. Lett. 2013, 4, 2061-2068.

(12) Hoy, J.; Morrison, P. J.; Steinberg, L. K.; Buhro, W. E.; Loomis, R. A. Excitation Energy Dependence of the Photoluminescence Quantum. J. Phys. Chem. Lett. 2013, 4, 2053-2060.

(13) García-Santamaría, F.; Chen, Y.; Vela, J.; Schaller, R. D.; Hollingsworth, J. A.; Klimov, V. I. Suppressed Auger Recombination in "Giant" Nanocrystals Boosts Optical Gain Performance. Nano Lett. 2009, 9, 3482-3488.

(14) Kazes, M.; Lewis, D. Y.; Ebenstein, Y.; Mokari, T.; Banin, U. Lasing from Semiconductor Quantum Rods in a Cylindrical Microcavity. Adv. Mater. 2002, 14, 317-321.

(15) Dang, C.; Lee, J.; Breen, C.; Steckel, J. S.; Coe-Sullivan, S.; Nurmikko, A. Red, Green and Blue Lasing Enabled by Single-exciton Gain in Colloidal Quantum Dot Films. Nat. Nano. 2012, 7, 335-339.

(16) Chen, Y.; Guilhabert, B.; Herrnsdorf, J.; Zhang, Y.; Mackintosh, A. R.; Pethrick, R. a.; Gu, E.; Laurand, N.; Dawson, M. D. Flexible Distributed-feedback Colloidal Quantum Dot Laser. Appl. Phys. Lett. 2011, 99, 241103.

(17) Chan, Y.; Caruge, J.-M.; Snee, P. T.; Bawendi, M. G. Multiexcitonic Two-state Lasing in a CdSe Nanocrystal Laser. Appl. Phys. Lett. 2004, 85, 2460. 
(18) Chan, Y.; Steckel, J. S.; Snee, P. T.; Caruge, J.-M.; Hodgkiss, J. M.; Nocera, D. G.; Bawendi, M. G. Blue Semiconductor Nanocrystal Laser. Appl. Phys. Lett. 2005, 86, 073102.

(19) Jasieniak, J. J.; Fortunati, I.; Gardin, S.; Signorini, R; Bozio, R.; Martucci, A.; Mulvaney, P. Highly Efficient Amplified Stimulated Emission from CdSe-CdS-ZnS Quantum Dot Doped Waveguides with Two-Photon Infrared Optical Pumping. Adv. Mater. 2008, 20, 69-73.

(20) Todescato, F.; Fortunati, I.; Gardin, S.; Garbin, E.; Collini, E.; Bozio, R.; Jasieniak, J. J.; Giustina, G. Della; Brusatin, G.; Toffanin, S.; et al. Soft-Lithographed Up-Converted Distributed Feedback Visible Lasers Based on CdSe-CdZnS-ZnS Quantum Dots. Adv. Funct. Mater. 2012, 22, 337-344.

(21) Signorini, R.; Fortunati, I.; Todescato, F.; Gardin, S.; Bozio, R.; Jasieniak, J. J.; Martucci, A.; Giustina, G. D.; Brusatin, G.; Guglielmi, M. Facile Production of Up-converted Quantum Dot Lasers. Nanoscale 2011, 3, 4109-13.

(22) Zhang, C.; Zhang, F.; Zhu, T.; Cheng, A.; Xu, J.; Zhang, Q.; Mohney, S. E.; Henderson, R. H.; Wang, Y. A. Two-photon-pumped Lasing from Colloidal Nanocrystal Quantum Dots. Opt. Lett. 2008, 33, 2437-9.

(23) Larson, D. R.; Zipfel, W. R.; Williams, R. M.; Clark, S. W.; Bruchez, M. P.; Wise, F. W.; Webb, W. W. Water-Soluble Quantum Dots for Multiphoton Fluorescence Imaging in Vivo. Science 2003, 300, 1434-1436.

(24) Zheng, Q.; He, G. S.; Prasad, P. N. A Novel Near IR Twophoton Absorbing Chromophore: Optical Limiting and Stabilization Performances at an Optical Communication Wavelength. Chem. Phys. Lett. 2009, 475, 250-255.

(25) Xing, G.; Liao, Y.; Wu, X.; Chakrabortty, S.; Liu, X.; Yeow, E. K. L.; Chan, Y.; Sum, T. C. Ultralow-Threshold Two-Photon Pumped Amplified Spontaneous Emission and Lasing from Seeded CdSe/CdS Nanorod Heterostructures. ACS Nano 2012, 6, 10835-10844.

(26) Piryatinski, A.; Ivanov, S. A.; Tretiak, S.; Klimov, V. I. Effect of Quantum and Dielectric Confinement on the Exciton-Exciton Interaction Energy in Type II Core/Shell Semiconductor Nanocrystals. Nano Lett. 2007, 7, 108-115.

(27) Nanda, J.; Ivanov, S. A.; Htoon, H.; Bezel, I.; Piryatinski, A.; Tretiak, S.; Klimov, V. I. Absorption Cross Sections and Auger Recombination Lifetimes in Inverted Core-Shell Nanocrystals: Implications for Lasing Performance. J. Appl. Phys. 2006, 99, 034309.

(28) Balet, L. P.; Ivanov, S. A.; Piryatinski, A.; Achermann, M.; Klimov, V. I. Inverted Core/Shell Nanocrystals Continuously Tunable Between Type-I and Type-II Localization Regimes. Nano Lett. 2004, 4, $1485-1488$.

(29) Cooney, R.; Sewall, S.; Sagar, D.; Kambhampati, P. Gain Control in Semiconductor Quantum Dots via State-Resolved Optical Pumping. Phys. Rev. Lett. 2009, 102, 127404.

(30) Saba, M.; Minniberger, S.; Quochi, F.; Roither, J.; Marceddu, M.; Gocalinska, A.; Kovalenko, M. V.; Talapin, D. V.; Heiss, W.; Mura, A.; et al. Exciton-Exciton Interaction and Optical Gain in Colloidal CdSe/CdS Dot/Rod Nanocrystals. Adv. Mater. 2009, 21, 4942-4946.

(31) Oron, D.; Kazes, M.; Banin, U. Multiexcitons in type-II Colloidal Semiconductor Quantum Dots. Phys. Rev. B 2007, 75, 035330.

(32) Park, Y.-S.; Malko, A.; Vela, J.; Chen, Y.; Ghosh, Y.; GarcíaSantamaría, F.; Hollingsworth, J.; Klimov, V. I.; Htoon, H. Near-Unity Quantum Yields of Biexciton Emission from CdSe/CdS Nanocrystals Measured Using Single-Particle Spectroscopy. Phys. Rev. Lett. 2011, 106, 187401.

(33) Zhao, J.; Chen, O.; Strasfeld, D. B.; Bawendi, M. G. Biexciton Quantum Yield Heterogeneities in Single CdSe (CdS) Core (Shell) Nanocrystals and Its Correlation to Exciton Blinking. Nano Lett. 2012, 12, 4477-4483.

(34) Cihan, A. F.; Hernandez Martinez, P. L.; Kelestemur, Y.; Mutlugun, E.; Demir, H. V. Observation of Biexcitons in Nanocrystal Solids in the Presence of Photocharging. ACS Nano 2013, 7, 47994809.

(35) Caruge, J.-M.; Chan, Y.; Sundar, V.; Eisler, H.; Bawendi, M. G. Transient Photoluminescence and Simultaneous Amplified Sponta- neous Emission from Multiexciton States in CdSe Quantum Dots. Phys. Rev. B 2004, 70, 085316.

(36) Jasieniak, J.; Pacifico, J.; Signorini, R.; Chiasera, A.; Ferrari, M.; Martucci, A.; Mulvaney, P. Luminescence and Amplified Stimulated Emission in CdSe-ZnS-Nanocrystal-Doped $\mathrm{TiO}_{2}$ and $\mathrm{ZrO}_{2}$ Waveguides. Adv. Funct. Mater. 2007, 17, 1654-1662.

(37) Greytak, A. B.; Allen, P. M.; Liu, W.; Zhao, J.; Young, E. R.; Popović, Z.; Walker, B. J.; Nocera, D. G.; Bawendi, M. G. Alternating Layer Addition Approach to CdSe/CdS Core/Shell Quantum Dots with Near-Unity Quantum Yield and High On-Time Fractions. Chem. Sci. 2012, 3, 2028-2034.

(38) Haus, J. W.; Zhou, H. S.; Honma, I.; Komiyama, H. Quantum Confinement in Semiconductor Heterostructure Nanometer-Size Particles. Phys. Rev. B 1993, 47, 1359-1365.

(39) Nanda, J.; Ivanov, S. A.; Achermann, M.; Bezel, I.; Piryatinski, A.; Klimov, V. I. Light Amplification in the Single-Exciton Regime Using Exciton-Exciton Repulsion in Type-II Nanocrystal Quantum Dots. J. Phys. Chem. C 2007, 111, 15382-15390.

(40) Ivanov, S. A.; Nanda, J.; Piryatinski, A.; Achermann, M.; Balet, L. P.; Bezel, I. V.; Anikeeva, P. O.; Tretiak, S.; Klimov, V. I. Light Amplification Using Inverted Core/Shell Nanocrystals: Towards Lasing in the Single-Exciton Regime. J. Phys. Chem. B 2004, 108, $10625-10630$.

(41) Muller, J.; Lupton, J. M.; Lagoudakis, P. G.; Schindler, F.; Koeppe, R.; Rogach, A. L.; Feldmann, J.; Talapin, D. V; Weller, H. Wave Function Engineering in Elongated Semiconductor Nanocrystals with Heterogeneous Carrier Confinement. Nano Lett. 2005, 5, 20442049.

(42) Sitt, A.; Sala, F. D.; Menagen, G.; Banin, U. Multiexciton Engineering in Seeded Core/shell Nanorods: Transfer from Type-I to Quasi-Type-II Regimes. Nano Lett. 2009, 9, 3470-3476.

(43) Li, J. J.; Wang, Y. A.; Guo, W.; Keay, J. C.; Mishima, T. D.; Johnson, M. B.; Peng, X. Large-Scale Synthesis of Nearly Monodisperse CdSe/CdS Core/Shell Nanocrystals Using Air-Stable Reagents via Successive Ion Layer Adsorption and Reaction. J. Am. Chem. Soc. 2003, 125, 12567-12575. 